\title{
ARQUEOLOGÍA Y MEMORIA DE LOS CAMINANTES DE LA PRECORDILLERA DE CAMARONES, SIERRA DE ARICA
}

\author{
ARCHAEOLOGY AND MEMORY OF THE TRAVELLERS \\ FROM THE HIGHLANDS OF CAMARONES, ARICA REGION
}

\author{
Magdalena García B.* y Rolando Ajata L.**
}

\begin{abstract}
En este trabajo se sintetizan los primeros resultados de una investigación centrada en la movilidad prehispánica y tradicional del área Centro Sur Andina, la cual toma como caso de estudio los caminos troperos que confluyen en la precordillera de Camarones. A diferencia de los asentamientos abandonados durante el siglo XVI, la red vial prehispánica continuó en uso hasta tiempos recientes, formando parte activa de los circuitos de movilidad de las comunidades que actualmente habitan estos espacios. En el recorrido a pie junto al arriero Manuel Viza (Illapata) por dos de estas rutas, a saber Pachica-Guañacagua e Illapata-Caritaya, relevamos 42 sitios arqueológicos que evidencian ocupaciones que datan por lo menos desde el periodo Intermedio Tardío (siglo X) hasta tiempos recientes (siglo XX). Tanto las rutas como algunos de los sitios registrados fueron reconocidos e incluso ocupados por los pobladores que participaron en los talleres realizados en los pueblos. Para muchos de ellos, los caminos constituyen un objeto de memoria y de conexión con el pasado que muchas veces no es posible expresar desde el lenguaje hablado. Con todo, en este trabajo concluimos que hasta la segunda mitad del siglo XX coexistieron diversas modalidades de tránsito a pie vinculadas a prácticas sociales diferenciadas, las que nos permitieron aproximarnos a los circuitos y lugares de origen de los caminantes.
\end{abstract}

Palabras claves: Caminantes, caminos troperos, quebrada de Camarones, movilidad, territorio.

This article presents a case study about prehispanic and traditional mobility in the South Central Andes, which centers around paths that converge on the foothills of the Camarones Valley. Unlike the prehispanic towns abandoned during the 16th century, this network of roads continued to be used until recent times, being part of active mobility circuits for the communities that currently inhabit these spaces. For many of them, the paths constitute an object of memory and connection to the past that cannot be accomplished through spoken language. Following two of these paths by foot allowed us to document a total of 42 archeological sites that show evidence of occupations dating back to the Late Intermediate period until recent times (centuries X-XX). The paths as well as certain sites were recognized and occupied by the people who participated in our ethnographic work. From this we are able to conclude that until the second half of the 20th century different strategies of transit coexisted, associated with diverse social practices that allowed us to identify travelers, their circuits and their places of origin.

Key words: Travelers, paths, Camarones valley, mobility, territory.

\section{Introducción}

Desde la arqueología, la movilidad poblacional en los Andes ha sido comprendida como una estrategia de adaptación a una geografía caracterizada por paisajes contrastantes y enclaves productivos rodeados por grandes extensiones de desiertos y montañas. A partir de esta idea, el caso de estudio que abordamos a continuación busca contribuir a demostrar que el fenómeno social de la movilidad encierra un proceso complejo, donde convergen simultáneamente varios intereses y prácticas sociales más allá de la complementariedad ecológica y el intercambio de objetos.

En el presente, los caminos troperos que confluyen en el curso superior de la quebrada de
Camarones, sierra meridional de Arica, no solo nos permiten recorrer minuciosamente este espacio geográfico, sino también viajar hacia el pasado, ya que a diferencia de los asentamientos prehispánicos abandonados durante la invasión hispana, los caminos troperos continuaron en uso hasta tiempos recientes, formando parte activa de los circuitos de movilidad de las comunidades que actualmente ocupan estos espacios, cuyas residencias se ubican adyacentes a los poblados prehispánicos.

La investigación sugiere que los recorridos por los caminos posibilitan un acto de memoria que no es posible desde el lenguaje hablado. En este sentido, estos constituyen un archivo que el viajero sabe leer y que lo conecta con el pasado. Este archivo se fue construyendo sincrónica y diacrónicamente a partir

\footnotetext{
* Universidad Católica del Norte, Instituto de Arqueología y Antropología. Centro de Investigación del Hombre en el Desierto (CIHDE). Arica, Chile. Correo electrónico: manegarciab@yahoo.com

** Universidad de Tarapacá-Universidad Católica del Norte. Arica, Chile, Programa de Magister en Antropología. Correo electrónico: rolandoajata@gmail.com
} 
de la acumulación de topónimos, ocupaciones y prácticas que remiten a ideas, significados, intensiones y experiencias atadas a una tradición y una historia. Por consiguiente, los caminos son también una expresión de territorialidad, entendiendo por esta la dimensión cultural que envuelve la relación de las comunidades con la tierra, proporcionando un sustrato para articular y contextualizar los sitios arqueológicos y lugares que desde nuestra perspectiva existían dispersos en la geografía.

La elección del curso superior de la quebrada de Camarones como centro (Figura 1) no es más que una construcción nuestra con fines operativos, ya que en realidad los caminos estudiados constituyen solo un pequeño segmento de la extensa y compleja red vial que articuló el área Centro Sur Andina desde tiempos prehispánicos hasta avanzado el siglo XX.
En efecto, su antigüedad se manifiesta en ramales que se dirigen directamente hacia asentamientos arqueológicos que datan del siglo $\mathrm{X}$, así como en la presencia de restos materiales de esa misma época (Cf. Muñoz et al. 1987; Muñoz y Briones 1996).

Salvo algunas excepciones (Muñoz y Briones 1996; Santoro et al. 2010; Valenzuela et al. 2011), la arqueología de Arica ha prestado poca atención a los caminos troperos, centrándose más bien en los asentamientos y su dinámica interna (Niemeyer et al. 1971; Dauelsberg 1983; Santoro et al. 2004; Muñoz y Chacama 2006). Y si bien estos autores han reconocido niveles de integración e interrelación entre las diferentes quebradas, los asentamientos continúan visualizándose como entidades independientes distribuidos como islas en la precordillera andina, pasando por alto la red vial que los comunica.

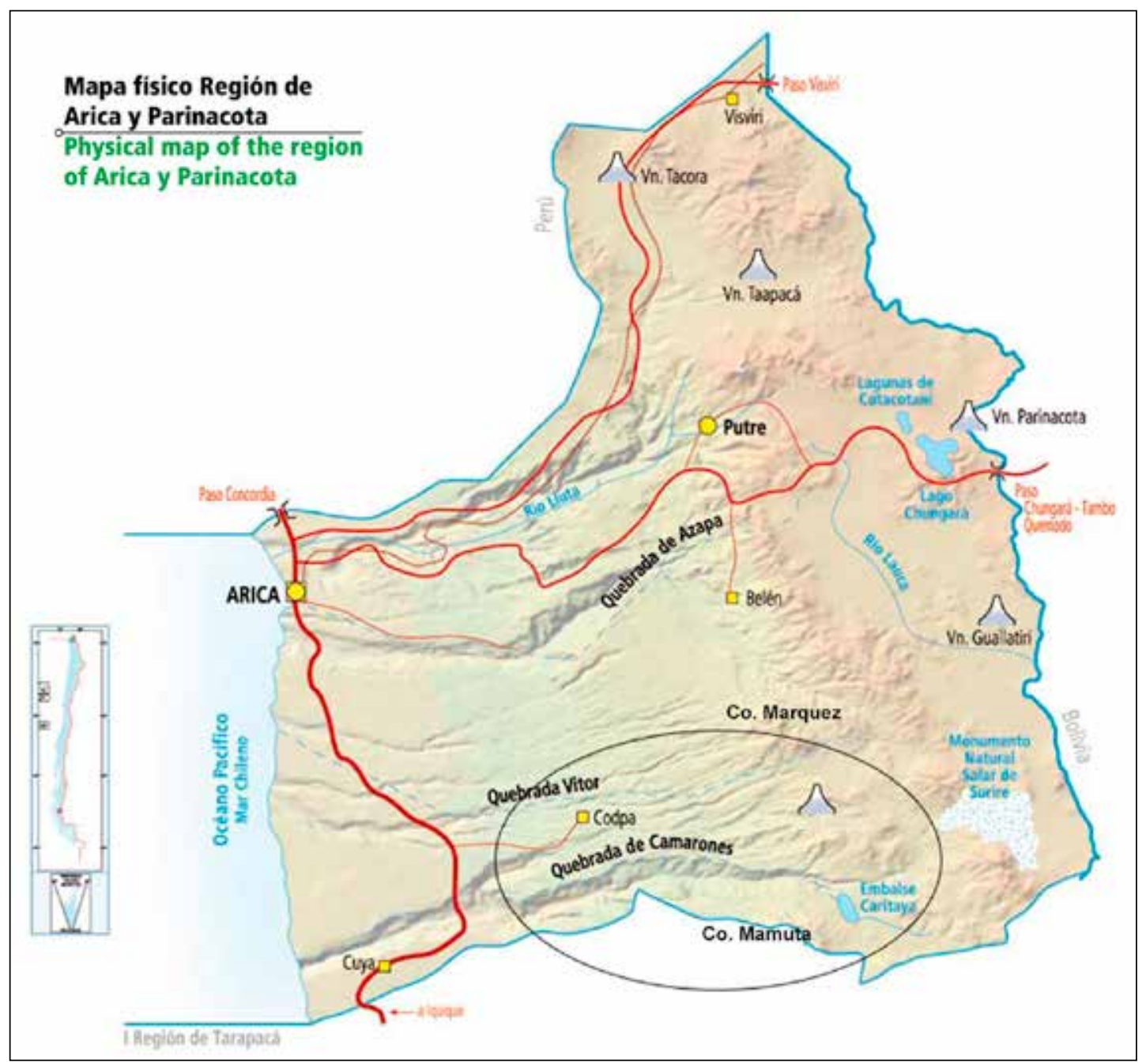

Figura 1. Ubicación del área de estudio en la región de Arica y Parinacota, Norte Grande de Chile. 


\section{Cómo abordamos a los caminantes}

El registro se realizó durante el 2011, a partir de actividades que tuvieron distintos niveles de planificación. Por una parte se recorrieron a pie los caminos que comunican la quebrada de Camarones con el valle de Codpa (ruta Pachica-Guañacagua) y con el altiplano de Caritaya (ruta Illapata-Caritaya). Estos recorridos fueron realizados en compañía del poblador Manuel Viza, de Illapata, quien se desempeñó como guía y arriero. Por otra parte se realizaron tres talleres abiertos a la comunidad en los pueblos de Illapata y Guañacagua, además de un focus group con los estudiantes del Liceo de Codpa. En los talleres se reprodujo de manera íntegra la trayectoria de las rutas caminadas a través de la proyección de fotografías y videos, buscando con ello la posibilidad de efectuar un viaje virtual con los asistentes. Se puso especial énfasis en las imágenes del sendero mismo, de los contextos arqueológicos asociados y los hitos geográficos sobresalientes del paisaje. Estas motivaron la participación activa de algunos pobladores, especialmente de aquellos de mayor edad que fueron activos caminantes en las décadas anteriores. Destacamos particularmente el reconocimiento que hicieron de las apachetas, majadas y paraderos dentro del universo de sitios arqueológicos registrados. Concluida la proyección, se trabajó sobre mapas impresos con diferentes escalas y tamaños -plotter e impresiones corrientes-, a los que los pobladores fueron agregando nuevas rutas troperas y topónimos que permitieron ir llenando de contenido un mapa oficial que inicialmente estaba en gran medida vacío.

El diseño de prospección arqueológica se realizó sobre la base de las metodologías implementadas en el área Circumpuneña, donde la arqueología de caminos tiene un desarrollo sustancialmente mayor (Aldunate et al. 2003; Berenguer 2004; Nielsen 2006; Núñez y Nielsen 2011; Pimentel 2009, 2011; Pimentel et al. 2011). Basándonos en estas, definimos dos unidades de análisis complementarias: el sendero y los sitios arqueológicos asociados. Por una parte los senderos fueron completamente georreferenciados a partir del reconocimiento de "puntos de sendero", los que no estuvieron más distantes que $500 \mathrm{~m}$. Por otra parte se registró la totalidad de los sitios prehispánicos, históricos y subactuales emplazados hasta $50 \mathrm{~m}$ a cada lado del eje de la vía. El registro de cada sitio se hizo utilizando una ficha estándar donde fue consignada su ubicación, emplazamiento, UTM, extensión, arquitectura, cronología relativa y caracterización de los materiales culturales en superficie a base de aspectos cronológicos, tipológicos y funcionales.

Por último, se llevó a cabo un mapeo completo de los senderos y de los sitios registrados por medio de sistemas de información geográfica, utilizando los softwares Google Earth y ArcGIS.

\section{Los caminos troperos identificados}

\begin{abstract}
iiiEs una carretera internacional!!! Claro, porque de aquí arriba [Esquiña] un desvío se va a Saguara, de Saguara pasa a Sucuna y de ahí ya directo a Parcohaylla. Y de Parcohaylla ya se puede pasar al otro lado de la frontera. Los que bajaban a Esquiña y subían a la pampa de Caritaya, esos iban allá a Mulluri, y el que quería ir a Camiña, de Caritaya hay un desvío a Camiña. Entonces es muy grande esto (...) por el trueque que existía antes, generalmente están todos los caminos conectados, todos los pueblos se conectan unos con otros (Manuel Viza, Illapata).
\end{abstract}

Hasta la fecha hemos logrado identificar siete rutas que confluyen en el curso superior de la quebrada de Camarones desde distintas direcciones (Figura 2). Como da cuenta el testimonio anterior, los destinos que se dan a conocer a continuación no constituyen el punto terminal de las rutas, sino puntos intermedios para acceder a otros destinos que por ahora no hemos precisado mayormente.

Las siete rutas identificadas son las siguientes:

Ruta 1. Desde Pachica en dirección norponiente hacia el valle de Codpa (longitud $28 \mathrm{~km}$ ).

Ruta 2. Desde Pachica rumbo nororiente hacia Sucuna (18 km), continuando hacia Ayco (24 $\mathrm{km})$ y Umirpa (32 km).

Ruta 3. Desde Esquiña rumbo norte hacia Saguara $(8 \mathrm{~km})$.

Ruta 4. Desde Illapata en dirección nororiente hacia Parcohaylla $(35 \mathrm{~km})$.

Ruta 5. Desde Illapata en dirección surponiente a Miñe Miñe $(40 \mathrm{~km})$.

Ruta 6. Desde Illapata en dirección sur a Nama (32 km). 


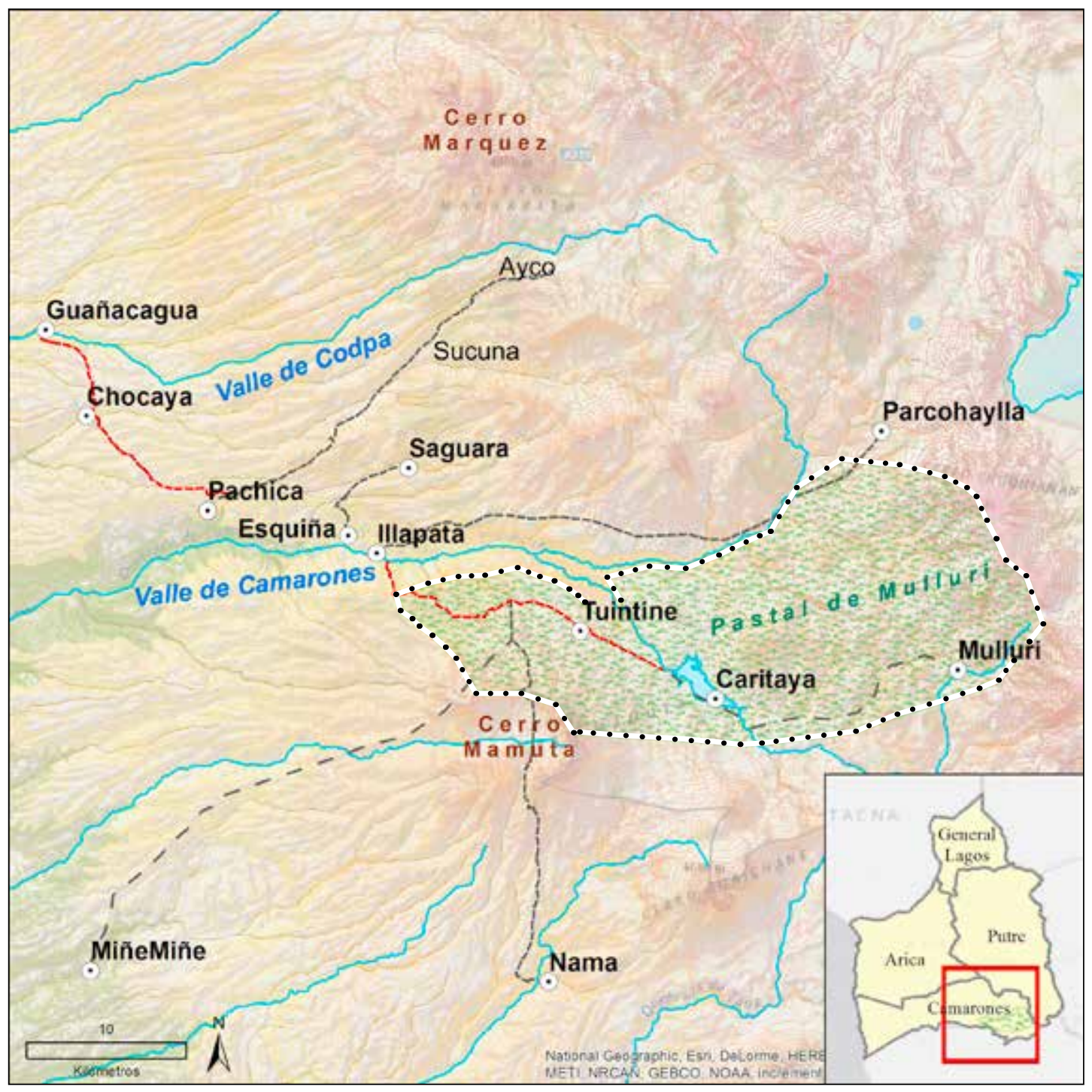

Figura 2. Los caminos troperos identificados que confluyen en el curso superior de la quebrada de Camarones, destacando en color rojo los caminos prospectados a pie. Con línea punteada se representa la extensión aproximada del pastal de la comunidad de Mulluri.

Ruta 7. Desde Illapata en dirección suroriente hacia Caritaya $(25 \mathrm{~km})$ continuando a Amuyo $(31 \mathrm{~km})$ y Mulluri $(45 \mathrm{~km})$, recorriendo la porción meridional del pastal de la comunidad de Mulluri.

En esta fase inicial de la investigación tenemos un conocimiento muy desigual de estos senderos, su trazado y estado de conservación. Solo las rutas 1 y 7 (segmento Illapata-Caritaya) fueron recorridas a pie y estudiadas sistemáticamente, mientras que las demás rutas han sido mapeadas total o parcialmente y documentadas con distinta intensidad desde el trabajo etnográfico.

\section{A la vera del camino. Los sitios arqueológicos registrados en las rutas Pachica-Guañacagua e Illapata-Caritaya}

El recorrido a pie permitió registrar 42 puntos con evidencias arqueológicas, 13 de ellos distribuidos en la ruta Illapata-Caritaya y 29 en la ruta Pachica-Guañacagua (Figura 3). En relación con la cronología, las ocupaciones registradas van 
desde el Intermedio Tardío a épocas subactuales (post-1973), siendo el 33\% de los sitios de carácter multicomponente, es decir, combinando ocupaciones pre y poshispánicas, el $26 \%$ de época indeterminada (estructuras sin asociación material), el 24\% monocomponente prehispánico y el $17 \%$ monocomponente histórico (Figura 4).

En relación a los tipos de sitios registrados, estos han sido agrupados en cinco categorías: tres categorías genéricas dadas por sitios habitacionales, sitios viales y petroglifos, y dos etnocategorías, majadas y paraderos. Estos últimos corresponden a sitios habitacionales y viales, respectivamente, no obstante han sido reconocidos y singularizados con esta denominación por los pobladores locales. Finalmente, la categoría petroglifos está compuesta por un solo sitio, del cual señalaremos únicamente que se emplaza en el descenso al pueblo de Guañacagua y que consiste en tres paneles donde se aprecia un camélido rectilíneo, figuras geométricas y múltiples motivos de cruces y calvarios de data colonial que se sobreponen a los primeros.

\section{Los sitios habitaciones}

Los sitios de carácter habitacional suman 17 , la mayoría de ellos emplazados en la ruta PachicaGuañacagua. Esto se explica ya que prácticamente

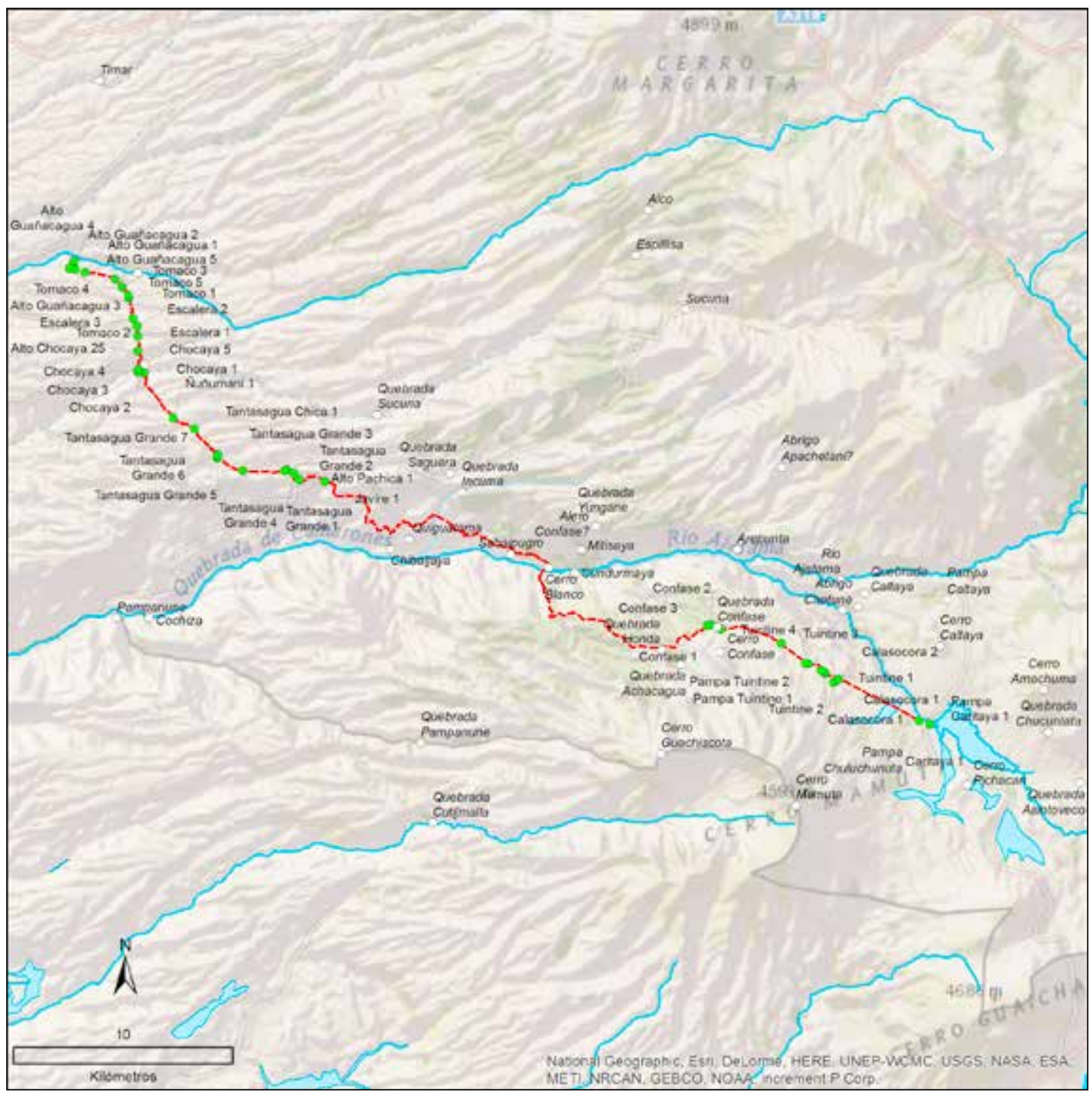

Figura 3. Distribución espacial de los 42 sitios arqueológicos registrados en la prospección. 


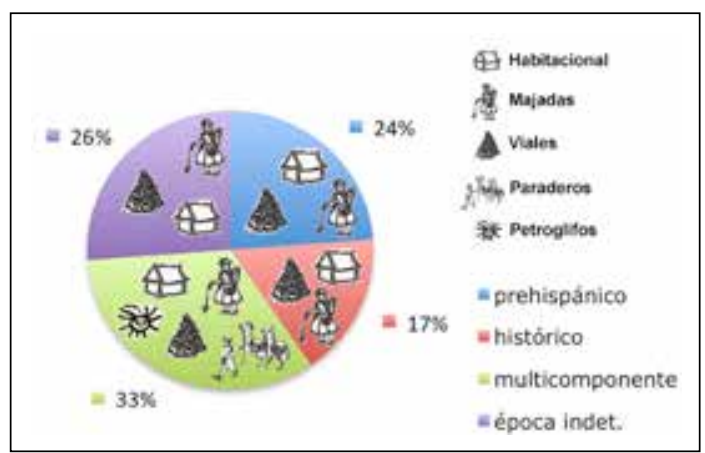

Figura 4. Cronología y tipo de sitios arqueológicos.

todos los sitios habitacionales de la ruta IllapataCaritaya se asocian a la categoría majadas. Los sitios habitacionales hacen referencia a contextos muy diversos que van desde simples parapetos hasta centros poblados densos como el pukara de Guañacagua.

El componente prehispánico se vincula a recintos pircados en general de hilada simple, con plantas circulares, ovales o irregulares, y tamaños variables entre $4 \mathrm{~m}^{2}$ y $56 \mathrm{~m}^{2}$. Asociado a estos encontramos cerámica alisada del tipo Charcollo, implementos de molienda, lascas de basalto, restos de maíz y algarrobo. Una mayor diversidad de tipos cerámicos se concentran en el pukara de Guañacagua (Ajata 2004). Asimismo, un contexto lítico más diverso se halló en uno de los sitios registrados en la quebrada de Chocaya, el que incluye dos puntas triangulares escotadas y una punta tetragonal de apariencia arcaica, entre otros materiales de data posterior.

Por su parte, el componente histórico está representado principalmente por vidrios, cerámica esmaltada, tarros de alimento, cuescos de durazno, herraduras y corrales con guano de cordero. Entre ellos destaca el campamento Caritaya, asociado a la construcción del tranque homónimo del año 1933. Está compuesto por ocho conjuntos arquitectónicos de adobe asociados a una alta densidad de materiales culturales, dispuestos en una superficie de 2 ha en el vértice surponiente del embalse.

\section{Las majadas}

Las majadas corresponden a residencias temporales que mantenían los pastores de la comunidad de Mulluri en las cabeceras de la quebrada de Camarones, las cuales eran ocupadas una vez que salía el pasto, durante los meses posteriores a las lluvias de verano. Este movimiento descendente con el ganado es conocido en la comunidad vecina de Isluga como costeo (Martínez 1989; Provoste 1976; Urrutia 2011).

Toda esas [majadas] se usaban una vez que salía el pasto, mayormente las usaba la gente que vivía en el pueblo de Mulluri por esos lados; cuentan que bajaban todos en el verano pa acá pa abajo con sus animales (...) Eran grandes las tropas (Manuel Viza, Illapata).

Diez sitios fueron identificados como majadas. En términos arqueológicos, estas consisten en recintos pircados a cielo abierto o junto a afloramientos rocosos o bloques aislados aprovechados como aleros. Las basuras domésticas registradas en las majadas permitieron discriminar sitios unicomponentes prehispánicos (4), sitios unicomponentes históricos (2) y sitios multicomponentes (3). Solo un caso quedó indeterminado por tratarse de una estructura sin asociación material.

El componente prehispánico está representado por recintos pircados simples y dobles que presentan plantas circulares, ovales o irregulares, con un tamaño promedio de $5 \mathrm{~m}^{2}$. Tanto al interior como al exterior de los recintos se registraron fragmentos de cerámica alisada, morteros cónicos, morteros planos, instrumentos de talla bifacial y desechos en materias primas silíceas, riolita, basalto y cuarzo. Destaca un sitio en pampa Caritaya, donde registramos una mayor diversidad cerámica, incluyendo un fragmento con pintura roja, fragmentos de un plato con base reforzada y dos fragmentos con una banda pintada de color rojo y café en el borde, que recuerdan tipos Isluga (Mauricio Uribe comunicación personal 2011; Sanhueza y Olmos 1981). Asociado a estos hallamos un contexto lítico que podría referir a una ocupación en tiempos arcaicos.

En algunos casos, el componente anterior se asocia a materiales históricos dados por fragmentos de cerámica mayólica, guano de camélido y cordero, restos óseos, cuescos de durazno, vidrio, latas de alimentos, ollas de fierro, entre otros. Es significativo señalar que no encontramos sitios unicomponentes prehispánicos con presencia de corrales, los que sí están presentes en los sitios multicomponentes y unicomponentes históricos. Este patrón podría responder a que "los llamos son aparte, o sea que 
los llamos casi no los encerraban sino que se rodeaban cerca de la majada, esos corrales son pa los corderos" (Manuel Viza, Illapata).

Finalmente, destacamos dos majadas emplazadas en los márgenes este y oeste del Pedregal de Tuintine, adosadas al afloramiento rocoso. La primera se orienta al este mirando el cerro Choqueananta (5.590 $\mathrm{msm}$ ) y fue reconocida como majada Calasocora. Consiste en un conjunto de recintos que incluyen un alero pircado, estructuras a cielo abierto, corrales y una estructura mediana abovedada identificada como frigider para guardar carne y quesos, o como trampa para zorros. El sitio se asocia a tarros de alimento, una etiqueta de alcohol con el sello Zeller y Mozer. Santa Cruz, restos óseos y guano.

La segunda fue reconocida como Cueva de Tuintine (Figura 5). Consiste en una cueva pircada abierta al poniente, asociada a varias estructuras edificadas a cielo abierto de carácter habitacional, corrales y un alineamiento de bloques de baja altura cubiertos con lajas que recuerdan a las casitas documentadas en el altiplano boliviano por Girault (1958).
Asimismo, se hallaron desechos líticos dispersos por todo el sitio, algunos fragmentos de cerámica, tarros de alimentos, una olla de fierro, guano y vidrio, entre otros desechos. Este sitio ya había sido referido por Niemeyer y colaboradores (1971) como un sitio asociado a material prehispánico y reocupado hasta ese entonces por pastores contemporáneos.

Por ejemplo, la cueva de Tuintine ahí estuve yo con mi tía. En un costado estaban los corderos, en el otro costado nosotros. Como cuarenta corderitos tenía. Era en el mes de marzo, si mal no recuerdo, y volvimos en el mes de junio. En febrero partimos (...) era el tiempo de parto porque ya había llovido entonces pastito salía. Uno sacaba agua en peñas o rocas, son como pozos así, como estanques. Yo me imagino que en este tiempo [mayo] todavía hay cuando llueve, cuando no llueve se secan esos pocitos y se pierde el agua y ahí ya regresamos al pueblo (...) Comíamos maíz tostado, queso y cuando

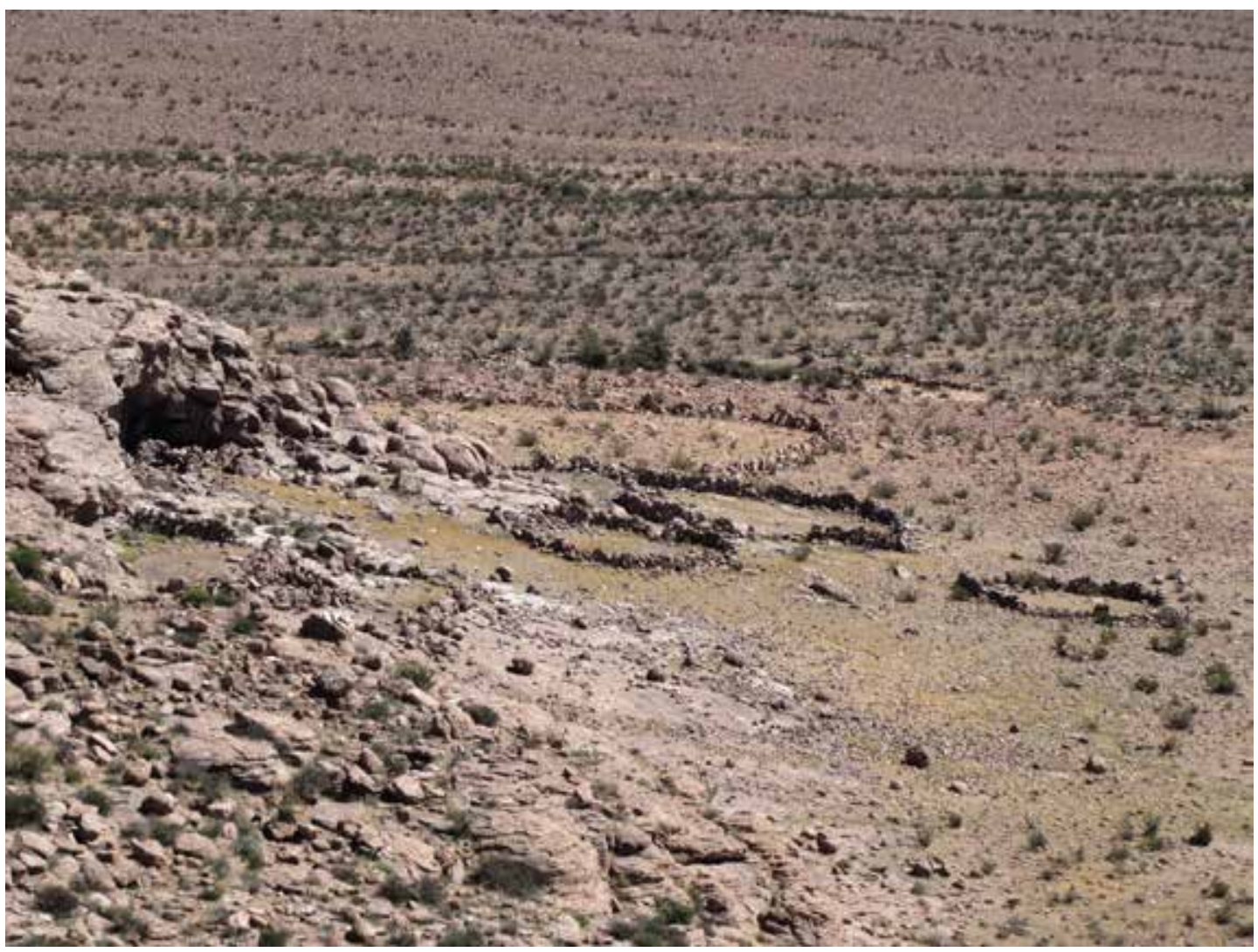

Figura 5. Cueva de Tuintine, ruta Illapata-Caritaya. 
faltaba se sacrificaba un corderito y después se hacía charqui y eso. Uno cocinaba con eso. Y como mi marido siempre nos iba a ver a la semana (...) llevaba choclo, papa, entonces no faltaba. Y de dormir dormíamos bien juntitos y cuando nos faltaba el pan, por eso digo que las piedras sirven para poder hacer las calatantas, uno prende el fuego nomás y pone sobre y después calatantas la da vuelta para allá para acá y después calienta en las bracitas y listo, queda rico (María Viza, nacida en Sora Sora y radicada en Guañacagua).

Como deja entrever el testimonio anterior, el movimiento descendente de los pastores a la precordillera incluía además relaciones de intercambio con los agricultores de las quebradas, de quienes obtenían maíz y fruta no solo para abastecer a las majadas durante el tiempo de pasto, sino también para llevar a sus pueblos en el altiplano. Estas relaciones de intercambio no solo involucraron a la comunidad de Mulluri, sino también a otras comunidades del altiplano Carangas.

Julio, agosto o septiembre ellos venían [a Esquiña], traían animales llamos para carnear y para hacer el trueque... ellos se llevaban el maíz, pero en grano sí, desgranado (...) venían hasta acá nomás... porque de aquí pa bajo, es que resulta que este maíz de acá para arriba es muy rico, es muy blando, de aquí para abajo ya no es este maíz, es más duro, es más desabrido, entonces la gente llegaba hasta aquí, hasta Pachica, de ahí se devolvían (...). Este maíz la gente venía a llevarse, pero no llevaban poco, llevaban 40, 50 llamos cargados. Cada llamo cargado 20 kilos, 30 kilos, ¿ese sabes pa cuándo? se cambiaba pa tiempo de lluvia, para pasar enero, febrero, marzo, porque en ese tiempo ya no pueden caminar por la lluvia. Eso lo hacían antes la gente por ejemplo de Guallatire, allá de Visvire, todo eso. De aquí a llegar a Guallatire por lo menos demoran tres días, para Bolivia es siete días, la gente todos los días arreando su llamito (...). También de Isluga venían para acá... de allá venían por Caritaya venían. De acá también pal lado de Mullure, Parcohaylla, toda esa gente venía para acá. Por ejemplo del lado de Bolivia, de Tambo Quemado al otro lado, ese se llama Chachacumane. Y ahí al frente hay otro pueblo que se llama Kosapa, esos son de una comunidad que se llama Turco (...) enfrente hay un pueblo que se llama Laguna, de Sajama, toda esa gente de Bolivia venía, y pa acá pal sur, por ejemplo, hay un pueblo que se llama Sacabaya y venían de otra parte que se llama Sabaya... Toda esa gente venía. Este tiempo [julio] están llegando de allá iitropas, tropas, todos los días!! Harta gente, tres, cuatro personas con tropas de cincuenta, sesenta animales; de Bolivia traían papas, de esa papa que hay allá en Belén (...) unas papas que revientan, que son ricas, harinosas, esas traían... traían cuarenta, cincuenta llamos cargados de papas, y de acá también se llevaban maíz (...) diferentes clases de papas, no una sola papa, hay otros son más duros, otros más bonitos según los colores, medios blancos, medios pintados, medios rojizos, así; pero aquí la gente llegaban a pelear, dos sacos, tres sacos, traían poco, pero había que repartir a todos iguales (...) Traían las frazadas, hay unos mantos que se tapaba acá, ellos traían talegas que los llamaban pa juntar pa maíz y así todo. Traían a veces también ropas, pantalones, en esos años ellos hacían, aquí la gente compraba, así como en el tiempo de los gentiles, por ejemplo unas chalecas, todo eso... de lana, de alpaca, de llamos así. Pero eso murió, nadie viene, y nadie va también. Esto van a ser ya como 15 años. Pa Codpa llegaban los mismos, porque en tiempo de fruta, como le digo yo, en aquellos años había harta fruta, hasta Bolivia se llevaban las peras, las uvas, los membrillos en febrero; por ser tienen la costumbre de bailar carnavales con membrillasos tirando... la gente como venía a llevar fruta de Codpa! (Eugenio Apata, nacido en Bolivia y radicado en Esquiña) (Figura 6).

\section{Los sitios viales}

Los sitios viales suman 12, entre ellos encontramos dos apachetas y 10 sitios con presencia de 


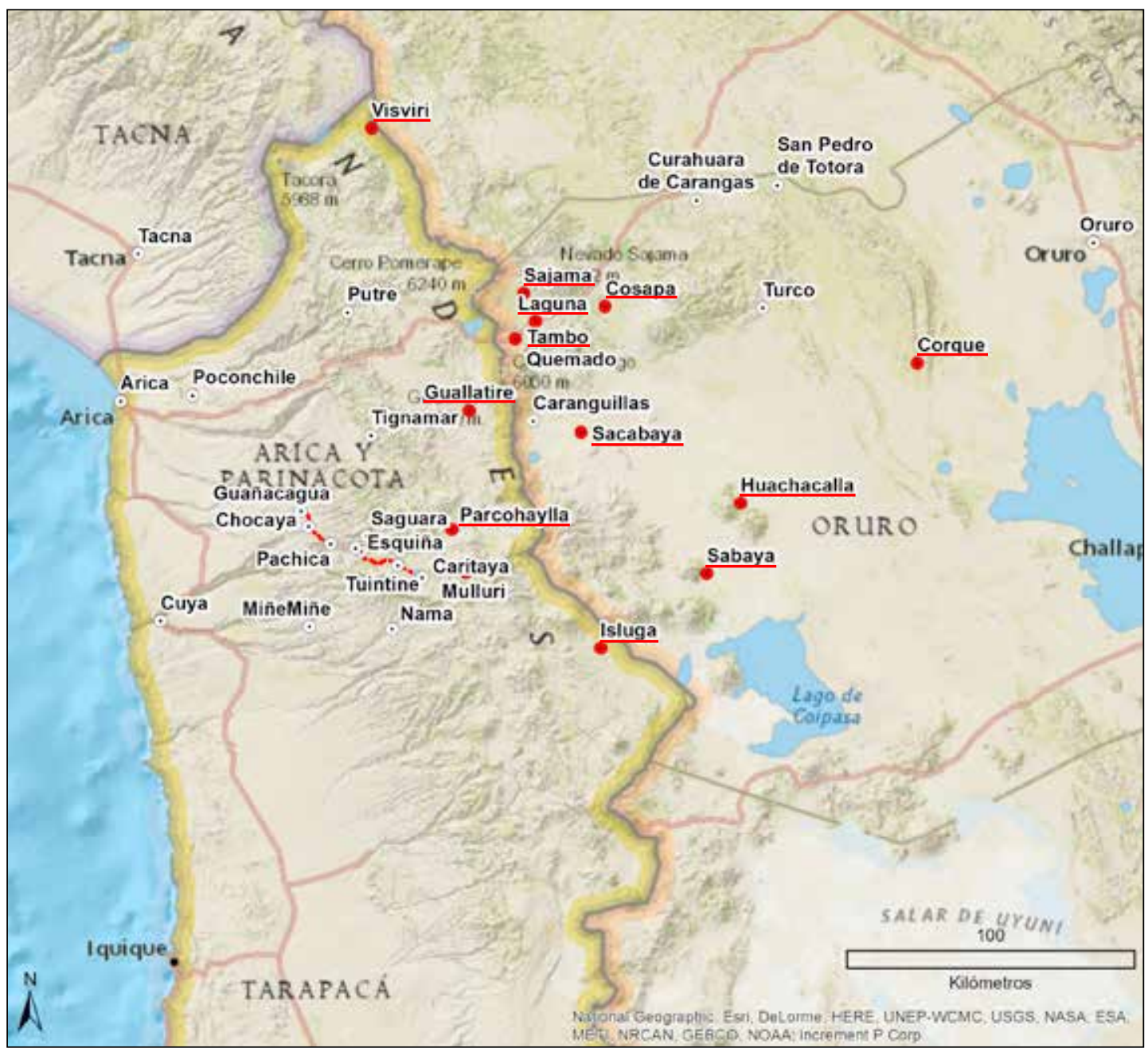

Figura 6. Subrayados, pueblos de origen de los pastores del altiplano Carangas que venían a buscar el maíz de Esquiña y la fruta y el vino de Codpa.

marcas o pininos. En relación con las primeras, la apacheta de Confase $(3.560 \mathrm{msm})$ se ubica en la ruta Illapata-Caritaya, en un punto de transición entre paisajes contrastantes, uno accidentado que caracteriza la geomorfología del borde sur de Camarones y otro llano propio de la estepa puneña. Asociado a ella encontramos únicamente desechos líticos prehispánicos, no obstante fue reconocida por los pobladores oriundos de Mulluri.

Por otra parte, la apacheta de Tantasagua (2.433 msm) se ubica en la ruta Pachica-Guañacagua y si bien pareciera no cumplir con los atributos propios de la apacheta andina -al estar bajo los $3.000 \mathrm{msm}$, no emplazarse en un abra o en un punto de transición de paisajes y poseer una altura menor a $1 \mathrm{~m}-$, fue reconocida como tal por los pobladores de Camarones. Entre otros, la apacheta parece estar marcando la bifurcación de ramales que continúan directo hacia Cochiza (SW) y a Pachica (SE). El montículo se asocia a fragmentos de cerámica alisada con y sin mica, fragmentos rojo engobados de posible filiación inka local, artefactos líticos en basalto, restos óseos, cuescos de durazno, loza y una herradura.

Bueno, antiguamente donde hay unas partes más altas por donde pasa el sendero, ahí amontonaban piedras. Cada viajero pasaba por ahí ... esas se llamaban A-P-A-CHE-T-A (...) tiene un significado, cuando uno va deja una piedra, ahí va por ejemplo todo el cansancio de uno o la enfermedad 
o cualquiera otra cosa ....antiguamente... escupían primero, pescaban una piedra y escupían tres veces (Eugenio Apata, nacido en Bolivia y radicado en Esquiña).

Hay que hacer "Madre Tierra no me agarre", tirarle la piedra limpiándote todo tu cuerpito, en las apachetas si. En las apachetas montón de piedras ahí. La piedrita límpiate tu cuerpito. Y después tírale al montón. Limpia tu cuerpo te vas sin mirar. Gracias a mi papá me enseñó a mí, no ves que viajaba ¡buuu! como le contaba por las pampas (Margarita Gaviño, nacida en Parcohaylla y radicada en Illapata).

A diferencia de las apachetas que se van construyendo de manera sucesiva a lo largo del tiempo lo que les permite a algunas de ellas alcanzar alturas notables, las marcas o pininos son construidas de una sola vez, utilizando bloques medianos a grandes y en general de un mismo tamaño. Las apachetas, en cambio, tienden a estar compuestas por piedras de diferentes tamaños, pero que puedan ser tomadas con una mano -para pasarla por el cuerpo y arrojarlaAsimismo, los pininos no necesariamente están emplazados en lugares de transición de paisajes ni en abras, aunque sí pueden estar demarcando bifurcaciones. Por cierto, tampoco tienen la connotación ritual de las apachetas.

Tienen la función de indicar la orientación correcta que debe seguir el viajero, especialmente cuando se enfrenta a espacios abiertos de horizonte lejano. Estas "markas" jalonan la mayoría de los senderos que atraviesan el desierto. Se encuentran de a una o pareadas, indicando algún cambio en la topografía: una cuesta o pendiente, el cruce de un antiguo aluvión, la bifurcación del sendero, etc (Muñoz y Briones 1996: 56).

La gente antigua ponía unas marcas, unos pininos, esos pininos los guiaban (...) pero no solamente acá, por ejemplo pa las quebradas en Pica, pa llá pa Macaya, pa Sibaya... Acá hay uno cuando pasamos [en vehículo], pero que está marcado con cemento (Ítalo Araya, Illapata).

En la ruta Illapata-Caritaya se registró un sitio con tres pininos a ambos lados del sendero. En la ruta Pachica-Guañacagua se registraron nueve, estos presentaron montículos variados en forma y tamaño, en general sin material asociado. En un caso, el pinino marca la bifurcación del sendero hacia Chitita y Guañacagua, donde además se construyó una estructura identificada como paraje para descansar.

\section{Los paraderos}

Los paraderos corresponden a puntos de confluencia de caminos troperos. Ambos casos registrados se ubican en la ruta Pachica-Guañacagua. Se trata de sitios complejos que presentan una amplia diversidad de estructuras, entre las que destaca una estructura principal de pirca doble reconocida como el hotel de los arrieros, además de recintos menores, parapetos, muros aislados o en $\mathrm{U}$, depresiones y amontonamientos, indistintamente asociados a material prehispánico, histórico y subactual. En particular, el paradero Cruce Chocaya o Cruce Corralones (Alto Chocaya 25 sensu Romero 2007) se asocia además a tres despejes circulares de 20 y $30 \mathrm{~m}$ de diámetro asociados a cerámica inka cuzqueña. El paradero se edificó en el borde norte de la quebrada de Chocaya donde confluye la ruta longitudinal serrana -a la que se asocia el segmento PachicaGuañacagua- con la huella que desciende desde el altiplano de Parcohaylla y que continúa en dirección a la costa (Figura 7). Constituyó el paradero de los agricultores de Esquiña hasta 1972, cuando llegó el camino vehicular al interior de la quebrada. Hasta allí acudían para sacar sus productos al camión que iba hacia Arica, que arribaba al paradero a las siete de la mañana. De esta manera, el sendero se transitaba de noche, demorando desde Esquiña-Illapata tres horas justas hasta Pachica y siete horas hasta este paradero. Pero no solo los agricultores de Esquiña llegaban a este punto, sino también los agricultores de Codpa, que iban a Chocaya a regar sus chacras, los pastores del altiplano adyacente y también los marchantes de Bolivia, que "traían charqui, sal, papas, coquita, copal, pululos, naranjas, llijllas hasta radio a pilas" (Margarita Gaviño, nacida en Parcohaylla y radicada en Illapata). "Llegaban al Cruce Corralones con carne seca, quinua, chuño, harina de maíz, sal. Mataban llamos en el lugar y cambiaban por fruta seca u orejones" (Víctor Ajata, nacido en Cobija y radicado en Arica).

Por su parte, el paradero de Alto Guañacagua (Ajata 2005) constituye el punto de confluencia 


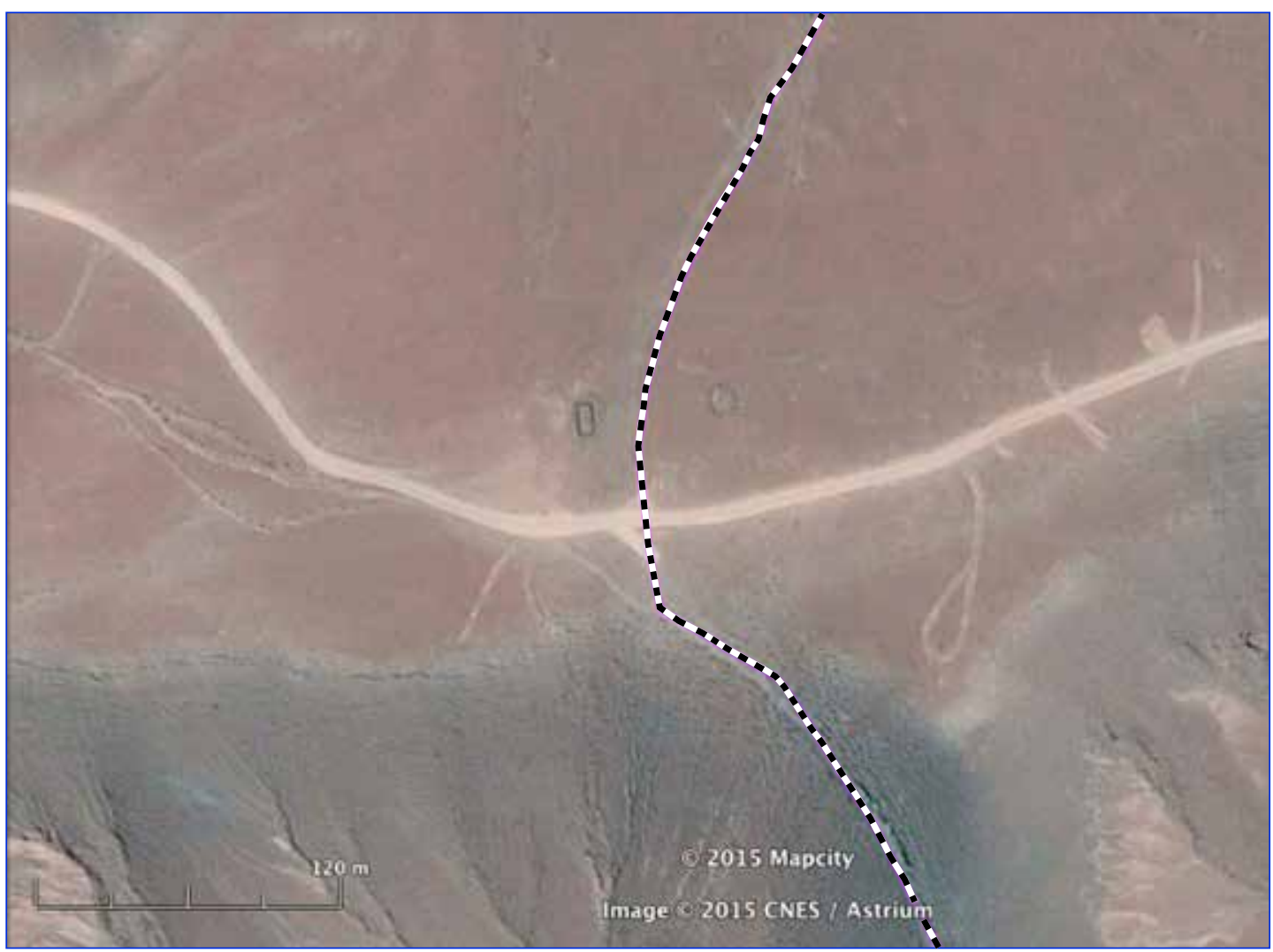

Figura 7. Vista satelital del paradero Cruce Chocaya o Cruce Corralones, destacando la estructura rectangular reconocida como el hotel de los arrieros. La ruta Pachica-Guañacagua se destaca con línea punteada (N-S) y sin colorear, la huella vehicular superpuesta al camino tropero que desciende desde Parcohaylla (E-W).

entre la ruta longitudinal serrana y el sendero que desciende desde los altos de Codpa el que continúa hacia el poniente. Estuvo operativo hasta la década del ochenta cuando se construyó el camino vehicular por el interior del valle de Codpa. Como en el caso anterior, los agricultores de Guañacagua subían allí para sacar sus productos a Arica, y fue también un punto de confluencia de personas que venían de lugares más lejanos, considerando la multiplicidad de recintos de construcción simple para el pernocte (p.e., muros en U, depresiones), reconocidos como "las carpas de antes" (Manuel Viza, Illapata).

\section{Conclusiones: identificación de los caminantes y sus modalidades de tránsito}

La cultura material registrada a la vera de los caminos, específicamente junto a las majadas, apachetas y paraderos, dan cuenta de un uso continuo de estos espacios por lo menos desde el Intermedio Tardío (siglo X) hasta avanzado el siglo XX. En este sentido es posible pensar en la existencia de un sistema de asentamiento (Aldunate et al. 1986) prehispánico que comparte elementos con el sistema histórico que hemos podido documentar arqueológica y etnográficamente.

Los usos etnográficos asociados a las rutas estudiadas hacen referencia a una memoria que data de la segunda mitad del siglo XX que, entre otros, hace referencia a distintas modalidades de tránsito que coexistían simultáneamente. Entre ellas se encuentran las diferentes dinámicas de trueque que vinculaban a las comunidades vecinas -como los agricultores precordilleranos y los pastores de Mulluri-, y otras dinámicas que incluían a comunidades más distantes, como los pastores del altiplano Carangas o los marchantes de Bolivia. Otra modalidad de tránsito se manifiesta en los movimientos que realizaban tanto agricultores como pastores hacia 
terrenos productivos alejados de las residencias principales (p.ej., chacras de Chocaya, pastos de la cabecera de Camarones). Además, debemos considerar el movimiento de los agricultores a los paraderos Cruce Corralones y Alto Guañacagua para sacar sus productos a Arica. Por último, se puede incluir también el movimiento de músicos y peregrinos a las festividades religiosas de Esquiña y Codpa, donde llegaban también pobladores de distintas comunidades del altiplano Carangas (p.ej., Huachacalla, Corque).

Finalmente, la memoria vinculada con estas modalidades permite comenzar a identificar y documentar a quienes transitaron por estas rutas durante la segunda mitad del siglo XX. A estos actores nos referimos genéricamente como agricultores de Camarones, agricultores de Codpa, pastores de Mulluri, pastores de otras comunidades del altiplano Carangas y marchantes de Bolivia. Cada uno de ellos hacía usos distintos de los caminos, con sus propios tiempos y circuitos, todo lo cual nos permite concluir que hasta por lo menos la década del setenta, cuando se cerraron las fronteras con Bolivia, coexistían distintos sistemas y modalidades de tránsito y tráfico que generaron movimientos en distintas direcciones, verticales y longitudinales, los que en última instancia permiten relativizar la visión altiplanocéntrica de la movilidad.

\section{Agradecimientos}

A los proyectos Fondecyt 1130279 y Fondart Regional 21117-6. La primera autora reconoce el patrocinio de la beca Conicyt Doctorado Nacional. Asimismo, agradecemos a los pobladores de Illapata, Esquiña, Guañacagua, Guatanave y Codpa por confiar en nosotros y compartir sus memorias dando cuerpo a esta investigación. Especialmente a Manuel Viza, Yolanda Challapa, Margarita Gaviño, Patricio Mamani, Emilia Araníbar, Silvia Challapa, Graciela Mamani, Augusto Mamani, Eugenio Apata, Lola Viza, María Viza, Víctor Ajata, Arnaldo Butrón y Francisco Rivera. Al profesor de la Escuela de Chitita Fernando Fernández y al alcalde de la I. Municipalidad de Camarones Iván Romero. Agradecemos también a Tania Muñoz, Charles Rees y Wilfredo Faúndez por su valiosa participación en la realización del trabajo de campo.

\section{Referencias Citadas}

Ajata, R.

2004 Congregación social y espacios públicos: pasado y presente en el valle de Codpa, norte de Chile. Boletín de la Sociedad Chilena de Arqueología 37: 7-17.

Ajata, R.

2005 Patrones de asentamiento prehispánico en el curso medioalto del valle de Codpa. Informe de Práctica Profesional en Arqueología, Universidad de Chile.

Aldunate, C., J. Berenguer, V. Castro, J. L. Martínez y C. Sinclaire 1986 Sobre la cronología del Loa Superior. Chungara 16-17: 333-346.

Aldunate, C., V. Castro y V. Varela

2003 Oralidad y arqueología: una línea de trabajo en las tierras altas de la región de Antofagasta. Chungara 35(2): 305-314.

Berenguer, J.

2004 Caravanas, interacción y cambio en el desierto de Atacama. Sirawi Ediciones, Santiago.

Bouysse-Cassagne, T. y J. Chacama

2012 Partición colonial del territorio, cultos funerarios y memoria ancestral en Carangas y precordillera de Arica (siglos XVI-XVII). Chungara 44(4): 669-689.

Dauelsberg, P.

1983 Investigaciones arqueológicas en la sierra de Arica, sector Belén. Chungara 11: 63-83.

Girault, L.

1958 Le culte des apacheta chez les Aymara de Bolivie. Journal de la Société des Américanistes 47: 33-46.
Martínez, G.

1989 Espacio y pensamiento I. Andes meridionales. Hisbol, La Paz.

Muñoz, I., J. Chacama y G. Espinosa

1987 El poblamiento prehispánico tardío en el valle de Codpa: una aproximación a la historia regional. Chungara 19: 7-61.

Muñoz, I. y L. Briones

1996 Poblados, rutas y arte rupestre precolombinos de Arica: descripción y análisis de sistema de organización. Chungara 28(1-2): 47-84.

Muñoz, I. y J. Chacama

2006 Complejidad social en las alturas de Arica: territorio, etnicidad y vinculación con el estado inca. Ediciones Universidad de Tarapacá, Arica.

Nielsen, A.

2006 Estudios internodales e interacción interregional en los Andes circumpuneños. En Esferas de interacción prehistóricas y fronteras nacionales modernas: los Andes Sur Centrales, editado por H. Lechtman, pp. 29-62. IEP-IAR, Lima.

Niemeyer, H., V. Schiappacasse e I. Solimano

1971 Padrones de poblamiento en la quebrada de Camarones (Prov. Tarapacá) (estudio preliminar del sector medio y superior del valle). Actas del VI Congreso de Arqueología Chilena, pp. 115-137, Santiago.

Núñez, L. y A. Nielsen

2011 Caminante, sí hay camino: reflexiones sobre el tráfico sur andino. En En ruta. Arqueología, historia y etnografía 
del tráfico surandino, editado por L. Núñez y A. Nielsen, pp. 83-110. Editorial Brujas, Córdoba.

Provoste, P. 1976. Antecedentes de la estructura socioeconómica de Isluga. Centro de Investigaciones Isluga, Universidad del Norte, Iquique.

Pimentel, G.

2011 Geoglifos e imaginarios sociales en el desierto de Atacama. En Temporalidad, interacción y dinamismo cultural. La búsqueda del hombre. Homenaje al profesor Lautaro Núñez Atencio, editado por A. Hubert, J. A. González y M. Pereira, pp. 163-200. Universidad Católica del Norte, Antofagasta.

Pimentel, G., C. Rees, P. de Souza y L. Arancibia

2011 Viajeros costeros y caravaneros. Dos estrategias de movilidad en el Período Formativo del Desierto de Atacama, Chile. En En ruta. Arqueología, historia y etnografía del tráfico surandino, editado por L. Núñez y A. Nielsen, pp. 43-81. Editorial Brujas, Córdoba.

Rivière, G.

1982 Sabaya, structures socio-économiques et représentations symboliques. Tesis Doctoral, École des Hautes Études en Sciences Sociales (EHESS).

Romero, A.

2007 Evaluación arqueológica y patrimonial en tramos vulnerables del proyecto de tendido eléctrico de Codpa y Camarones. Manuscrito en posesión del Consejo de Monumentos Nacionales.

Sanhueza, J. y O. Olmos

1981 Usamaya 1, cementerio indígena de Isluga, Altiplano de Iquique, I Región, Chile. Chungara 8: 169-207.

Santoro, C., A. Romero, V. Standen y A. Torres

2004 Continuidad y cambio en las comunidades locales, periodos Intermedio Tardío y Tardío, Valles Occidentales del área Centro Sur Andina. Chungara 36 (vol. esp. 1): 235-247.

Santoro, C., T. Dillehay, J. Hidalgo, D. Valenzuela, A. Romero, F. Rothhammer y V. Standen

2010 Revisita al tercer caso de verticalidad de John Murra en las costas de los Andes Centrales y Centro Sur. Chungara 42(1): 325-340.

Valenzuela, D., C. Santoro y L. Briones

2011 Arte rupestre, tráfico e interacción social: cuatro modalidades en el ámbito exorreico de los Valles Occidentales, norte de Chile (periodos Intermedio Tardío y Tardío, ca. 1000-1535 d.C.). En En ruta. Arqueología, historia y etnografía del tráfico surandino, editado por L. Núñez y A. Nielsen, pp. 199-245. Editorial Brujas, Córdoba.

Urrutia, F.

2011 Participación social en la quebrada de Camiña: entre los avatares de la tradición y la dinámica municipal. Memoria de Título en Antropología, Universidad de Chile. 
